\title{
Publicação dos trabalhos de Iniciação Científica da UFCG, Campus de Patos, PB
}

João Paulo Barbosa Anastácio*; João Nilton Lopes de Souza**; Rodrigo Araújo Rodrigues**; Rodrigo Alves Ribeiro**, Bárbara Vanessa de Brito Monteiro**

\footnotetext{
Graduado em Odontologia pela Universidade Federal de Campina Grande

** Professor da Universidade Federal de Campina Grande
}

\section{RESUMO}

Os programas de Iniciação Científica (IC) representam grande parte das pesquisas realizadas por estudantes de graduação. $\mathrm{O}$ objetivo desse estudo foi identificar a porcentagem de publicação destas pesquisas, realizadas nos cursos do Campus de Patos, $\mathrm{PB}$, aferir o tempo entre a apresentação da pesquisa no congresso de IC da instituição e sua publicação, identificar o curso e a área de origem da pesquisa. A partir da relação de alunos de IC nos anos de 2009 a 2014, foram consultados os dados das pesquisas nos Anais do Congresso de Iniciação Científica da Científica da Universidade Federal de Campina Grande, publicados no periódico Saúde \& Ciência Online. Dois pesquisa-dores realizaram buscas nas bases de dados Medline, LILACS e BBO, cruzando os dados dos resumos. O índice de publicação foi baixo, com $10,4 \%$ das pesquisas publicadas, sendo $10(30 \%)$ em periódicos Qualis A2. O tempo médio de publicação foi de 24 meses e o curso que obteve mais publicações no período estudado foi o de Medicina Veterinária. No curso de Odontologia, destacaram-se as áreas de Saúde Coletiva e Dentística. Apesar da relevância e das contribuições advindas das atividades de IC, ainda são poucos os levantamentos realizados sobre estas pesquisas e suas publicações.

Descritores: Formatos de Publicação. Pesquisa. Educação Superior. Odontologia.

\section{INTRODUÇÃO}

A produção científica brasileira, mensurada pelo número de artigos publicados em periódicos, apresentou significativa elevação percentual, se comparada a outros países ${ }^{1}$. O Conselho Nacional de Desenvolvimento Científico e Tecnológico (CNPq), agência pública que promove a Ciência, Tecnologia e Inovação, por meio da constituição e aperfeiçoamento de recursos humanos, direciona financiamentos aos projetos de pesquisa e, desde a década de 1990, tem fomentado a Iniciação Científica (IC), com a finalidade introduzir o estudante de graduação no mundo da pesquisa científica, estimulando novos talentos ${ }^{2}$.

O CNPq, ao estabelecer parcerias com instituições de ensino superior, criou o Programa Institucional de Bolsas de Iniciação Científica (PIBIC) e o Programa de Voluntários de Iniciação Científica (PIVIC) e as instituições conveniadas possuem parte da responsabilidade com os programas ${ }^{3}$.

O objetivo final de toda a pesquisa é sua publicação em periódicos científicos de qualidade, de maneira a difundir o conhecimento. Atualmente, no Brasil, os periódicos são categorizados pelo sistema 
Qualis, elaborado pela Coordenação de Aperfeiçoamento de Pessoal de Nível Superior (CAPES) e classificados em ordem decrescente de relevância: A1, A2, B1, B2, B3, B4, B5 e C $\mathrm{C}^{4-6}$.

Desta forma, os autores se propõem a analisar a publicação dos trabalhos desenvolvidos nos programas de IC no Campus de Patos, PB.

\section{METODOLOGIA}

O presente estudo é de caráter transversal e descritivo. Para sua realização foi utilizada, inicialmente, a relação de alunos pesquisadores nos programas PIVIC e PIBIC, nos anos de 2009 até 2014. A partir desta identificação, foram consultadas as respectivas pesquisas com base nos Anais do Congresso de Iniciação Científica da Universidade Federal de Campina Grande (UFCG), publicados no periódico Saúde \& Ciência Online. Para verificar se os resumos foram publicados em periódicos indexados, dois pesquisadores independentes e previamente calibrados realizaram buscas utilizando as bases de dados Medline, LILACS e BBO.

$\mathrm{O}$ protocolo de busca inicialmente realizado cruzou o nome do primeiro autor acompanhado de palavras-chave do título. Caso não fosse localizado, o nome do último autor também era incluído na busca. Em seguida algumas características dos resumos encontrados foram comparadas àquelas dos resumos publicados em anais do congresso de Iniciação Científica da UFCG, tais como autores, título, objetivos, metodologia (tamanho da amostra e atividades laboratoriais desenvolvidas), resultados e conclusões. Se após as avaliações dos dois examinadores o resumo encontrado no periódico estivesse de acordo com os itens descritos, a pesquisa seria considerada como publicada.
Também foram avaliadas outras variáveis: tempo transcorrido entre a publicação dos Anais do Congresso de IC da UFCG e a publicação no periódico, curso de origem (Ciências Biológicas, Engenharia Florestal, Odontologia e Medicina Veterinária), área de pesquisa (nos trabalhos do Curso de Odontologia) e classificação do periódico onde o trabalho foi publicado, definida pelo Qualis/CAPES.

Os resultados são apresentados na forma de estatísticas descritivas.

\section{RESULTADOS E DISCUSSÃO}

Dos 317 trabalhos realizados na IC, apenas $33(10,4 \%)$ foram publicados. $\mathrm{Na}$ tabela 1 observa-se o número de trabalhos de acordo com o curso. Desse total, $83 \%$ se encontram na categoria PIBIC e praticamente a metade das pesquisas são oriundas do curso de Medicina Veterinária. Segundo informações da administração, o quadro docente do curso de Medicina Veterinária apresenta o maior número de professores doutores.

Com relação aos estudantes pesquisadores, $176(54,6 \%)$ são do gênero feminino e $146(43,4 \%)$ do gênero masculino. A maior prevalência de pesquisadoras está de acordo com o observado em outros estudos, como pesquisa realizada pela Fundação de Amparo à Pesquisa do Estado de São Paulo (FAPESP) ${ }^{7}$, a qual avaliou características dos pesquisadores em IC no período de 1987 a 2007, demonstrando que $51 \%$ deles, em cursos da área da saúde, eram do sexo feminino. Tal fato é de suma importância, pois estamos vivendo crescente "feminização" do acesso universitário e ao trabalho, fato comum em outros países, como os Estados Unidos da América ${ }^{8}$. Para o Brasil, tal processo deu-se em meados da década de 1990, com evidente aumento da participação feminina, principal- 
Tabela 1 - Frequência e percentual dos trabalhos de Iniciação Científica realizados na instituição e publicados, de acordo com o curso

\begin{tabular}{ccc}
\hline Curso & Trabalhos de Iniciação Científica & Publicações \\
& n (\%) & n (\%) \\
\hline Ciências Biológicas & $35(11 \%)$ & $1(0,31 \%)$ \\
Engenharia Florestal & $81(25,6 \%)$ & $5(1,5 \%)$ \\
Odontologia & $48(15,1 \%)$ & $6(1,8 \%)$ \\
Medicina Veterinária & $153(48,3 \%)$ & $21(6,6 \%)$ \\
TOTAL & $317(100 \%)$ & $33(10,4 \%)$ \\
\hline
\end{tabular}

mente nas atividades de ensino e saúde 9 . Contudo, a variável gênero não influenciou a taxa de publicação em estudo anterior ${ }^{10}$.

Analisando as pesquisas publicadas pelo curso de Odontologia, destacam-se as áreas de Saúde Coletiva e Dentística, seguidas pelas áreas de Farmacologia e Materiais Dentários. A classificação dos periódicos nos quais os trabalhos foram publicados consta da tabela 2.

Tabela 2 - Distribuição das publicações de acordo com a classificação Qualis/CAPES, considerando todos os cursos

\begin{tabular}{cc}
\hline $\begin{array}{c}\text { Classificação } \\
\text { Qualis/CAPES }\end{array}$ & $\begin{array}{c}\text { Publicações } \\
\text { n (\%) }\end{array}$ \\
\hline A2 & $10(30 \%)$ \\
B1 & $2(5,5 \%)$ \\
B2 & $1(3,7 \%)$ \\
B3 & $1(3,7 \%)$ \\
B4 & $6(18,2 \%)$ \\
B5 & $11(33,6 \%)$ \\
Não classificado & $2(5,5 \%)$ \\
TOTAL & $33(100 \%)$ \\
\hline
\end{tabular}

$\mathrm{Na}$ tabela 3 observa-se esta mesma avaliação, porém restrita aos trabalhos produzidos no curso de Odontologia, que iniciou suas atividades em 2012.
Tabela 3 - Distribuição das publicações de acordo com a classificação Qualis/CAPES, considerando o curso de Odontologia

\begin{tabular}{cc}
\hline $\begin{array}{c}\text { Classificação } \\
\text { Qualis/CAPES }\end{array}$ & $\begin{array}{c}\text { Publicações } \\
\text { n (\%) }\end{array}$ \\
\hline B3 & 1 \\
B4 & 1 \\
B5 & 3 \\
TOTAL & $\mathbf{5}$ \\
\hline
\end{tabular}

Em estudo de Matelli-Júnior et al. ${ }^{11}$ que avaliou a condição da pesquisa odontológica no Estado do Minas Gerais, demonstrou que as áreas de Dentística e Patologia Bucal estavam entre as mais pesquisadas. Outra análise de dados referentes aos trabalhos apresentados na $27^{\mathrm{a}}$ Reunião Anual da Sociedade Brasileira de Pesquisa Odontológica $(\mathrm{SBPqO})^{12}$ evidenciou que a maior taxa de pesquisas foi nas áreas de Odontologia Preventiva e Social e Materiais Odontológicos.

Com relação ao percentual de publicação, chegou-se ao resultado que 33 $(10,4 \%)$ dos trabalhos pesquisados foram publicados em periódicos e $6(1,9 \%)$ estão em fase de avaliação para posterior publicação. Tal resultado é inferior a outros estudos ${ }^{13,14}$, que concluíram que $19 \%$ a $52,2 \%$ das 
pesquisas realizadas são publicadas. Tal fato pode ser elucidado tanto pelas exigências de qualidade dos periódicos, pelo pequeno número de periódicos se comparado ao número de trabalhos, pela pouca qualidade metodológica da pesquisa, pela falta de compatibilidade entre a pesquisa e o escopo das revistas ${ }^{6}$. Algumas questões que dificultam a publicação das pesquisas em periódicos podem ser elucidadas pelo insuficiente financiamento para o setor de ciência e tecnologia, a pouca experiência dos alunos pesquisadores e também como reflexos das ainda poucas e disputadas vagas em programas de iniciação científica ${ }^{15}$.

Analisando o tempo entre a apresentação no congresso de IC e a publicação em periódicos, constatou-se que $36,4 \%$ das pesquisas levaram cerca de 24 meses para serem publicadas. Bergoli et al. ${ }^{5}$ constataram um tempo médio de 24,1 meses entre a apresentação em congressos e a publicação em periódicos. Esse resultado corrobora com o tempo médio de publicação dos trabalhos do presente estudo (24 meses). Alguns trabalhos registram um período de 24 a 60 meses para a publicação das pesquisas $^{16,17}$.

Analisando a relação Qualis/CAPES dos trabalhos publicados em periódicos, observa-se que $10(30,0 \%)$ das pesquisas estão em periódicos com classificação A2, sendo todas referentes ao curso de Medicina Veterinária.

\section{CONCLUSÕES}

Conclui-se que, de forma geral, a taxa de publicação dos trabalhos desenvolvidos no programa de IC do Campus de Patos, PB é baixa e que o curso que obteve mais publicações no período estudado foi o de Medicina Veterinária. Com relação ao curso de Odontologia, as áreas de Saúde coletiva e
Dentística tiveram mais trabalhos publicados em periódicos. Apesar da relevância e das importantes contribuições advindas das atividades de IC, ainda são poucos os levantamentos realizados sobre as devidas pesquisas e suas publicações. Assim, existe a necessidade de fomento ao desenvolvimento de investigações que venham a contribuir para a análise e um maior conhecimento sobre o tema.

\section{ABSTRACT \\ Publication of the studies from scientific initiation programs at UFCG - Campus de Patos, PB}

The Undergraduate Research Programs (URP) represent a large part of research conducted by undergraduate graduate students. The aim of this study was to identify the percentage of publication of this research, conducted in the Patos Campus courses, PB, measuring the time between the presentation of research on IC congress of the institution and its publication, identify the course and the area of research. From the URP students' records in the years 2009-2014, we consulted data from the Proceedings of the URP Congress at Federal University of Campina Grande (UFCG), published in the journal Saúde \& Ciência Online. Two researchers conducted searches of Medline, LILACS and $\mathrm{BBO}$, crossing the data from abstracts. The publication rate was low, with $10.4 \%$ of published research, $10(30 \%)$ in Qualis A2 journals. The mean time for publication was 24 months and the Veterinary Medicine course got more publications in the studied period. For Dentistry, the highlights were the in areas of Public Health and Operative Dentistry. Despite the relevance and contributions from the URP activities, there are few surveys on these studies and publications.

Descriptors: Publication Formats. Research. Education, Higher. Dentistry. 


\section{REFERÊNCIAS}

1. Nadanovsky P. Growth in Brazilian scientific output in public health dentistry. Cad Saúde Pública. 2006;22(5):886-7.

2. Conselho Nacional de Desenvolvimento Científico e Tecnológico (CNPq). [Acesso em 17 de março de 2015]. Disponível em : http://www.cnpq.br.

3. Reis L.A. Programa institucional de bolsas de iniciação científica do IBAMA: uma política de pesquisa. [Dissertação] Mestrado em Educação, Universidade Católica de Brasília; 2007.

4. Coordenação de Aperfeiçoamento de Pessoal de Nível Superior (Capes). [Acesso em 22 de fevereiro de 2015]. Disponível em: http://www.capes.gov.br.

5. Bergoli CD, Amaral M, Rosa RA, Ardenghi TM. Subsequent publication of abstracts presented at the Brazilian Society of Oral Research Meeting. Int J Dent. 2011;10(4): 200-3.

6. Walby A, Kely AM, Georgakas C. Abstract to publication ratio for papers presented at scientific meetings: how does emergency medicine compare? Emerg Med. 2001;13:460-4.

7. Cavalcante RA, Barbosa DR, Bonan PRF, Pires MBO, Martelli-Jr H. Perfil dos pesquisadores da área de odontologia no Conselho Nacional de Desenvolvimento Científico e Tecnológico (CNPq). Rev Bras Epidemiol. 2008; 11(1):106-13.

8. The Economist. Women in the workforce, January 2nd, 2010. [Acessado em 20 de outubro de 2015]. Disponível em: http://www.economist.com/node/ 15174418 .

9. Lavinas, L. Empregabilidade no Brasil: inflexões de gênero e diferenciais femininos. Texto para discussão $\mathrm{n}^{\circ} 826$. Rio de Janeiro, setembro de 2001. [Acessado em 20 de outubro de 2015]. Disponível em: http://www.en.ipea.gov. br/agencia/images/stories/PDFs/TDs/td $\underline{0826}$

10. Rostad K, Arkness DW. Publication rate expressed by age, gender and academic position - a large-scale analysis of Norwegian academic staff. J Informetrics. 2015;9(2):317-33.

11. Martelli-Júnior H, Vieira Júnior JR, Domingos MA, Barbosa DR, BritoJúnior M, Bonan PRF. Pesquisa odontológica no estado de Minas Gerais, Brasil: análise retrospectiva de 1986 a 2006. Arq Odontol. 2007;43:23-9.

12. Xavier AFC,Sílva ALÓ,Cavalcanti AL. Análise da produção científica em Odontologia no Nordeste brasileiro com base em um congresso odontógico. Arq Odontol. 2011;47(3):127-34.

13. Yolcu U, Ozcan A. Publication rates in peer-reviewed journals of abstracts presented at the Oral and Maxillofacial Surgery Society of Turkey meetings 2007-2012. Br J Oral Maxillofac Surg. 2015;(9):849-53.

14. Livas C, Pandis N, Ren Y.Full-text publication of abstracts presented at European Orthodontic Society congresses. Eur J Orthod. 2014; 36(5):569-75.

15. Lino MM, Backes VMS, Ferraz F, Reibnitz KS, Martini JS. Análise da produção científica em educação em enfermagem na região sul do Brasil. Texto Contexto Enferm, 2010;19(2):26573.

16. Roy D, Sankar V, Hughes JP, Jones A, Fenton JE. Publication rates of scientific papers presented at the Otorhinolarygological Research Society 
meetings. Clin Otolaryngol Allied Sci. 2001;26(3):253-6.

17. Alonso-Arroyo A, Aleixandre-Benavent A, Vidal-Infer A, Anguita-Sánchez M, Chorro-Gascó FJ, Bolaños-Pizarro $\mathrm{M}$ Castelló-Cogollos L, Navarro-Molina C, Valderrama-Zurián. Subsequent full publication of abstracts presented in the Annual Meeting of the Spanish Society of
Cardiology. Rev Esp Cardiol. 2014;67:15-21.

Correspondência para:

Rodrigo Alves Ribeiro

e-mail: rdrgalves@hotmail.com Universidade Federal de Campina Grande Av. Universitária, s/n - Jatobá 58708-110 Patos, PB 